\title{
Detection of ascaridoid nematode parasites in the important marine food-fish Conger myriaster (Brevoort) (Anguilliformes: Congridae) from the Zhoushan Fishery, China
}

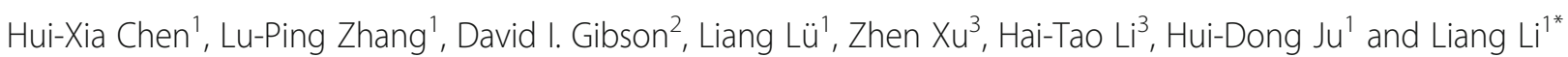

\begin{abstract}
Background: The whitespotted conger Conger myriaster (Brevoort) (Anguilliformes: Congridae) is an extremely marketable food fish, commonly consumed as sashimi or sushi in some Asian countries (i.e. Japan, Korea and China). Conger myriaster is also suspected as being an extremely important source of human anisakidosis. However, there is currently very little information on the levels of infection with ascaridoid nematode parasites in this economically important marine fish. The aims of the present study are to determine the species composition, prevalence and mean intensity of ascaridoid parasites of C. myriaster caught in the Zhoushan Fishery.

Results: A total of 1142 third-stage ascaridoid larvae were isolated from 204 C. myriaster. The overall prevalence of infection was 100\% (mean intensity 5.6). Nine species of such larvae were accurately identified using integrative taxonomic techniques involving both morphological and genetic data; these included Anisakis pegreffii, A. typica and $A$. simplex (sensu stricto) $\times$ A. pegreffii, Hysterothylacium fabri, $H$. aduncum, $H$. sinense, $H$. amoyense, $H$. zhoushanense and Raphidascaris lophii. Although high levels of infection and species richness were revealed in $C$. myriaster, most of the ascaridoid parasites (1135 individuals) were collected from the body cavity and visceral organs of the fish and only seven individuals of $A$. pegreffii were found in the musculature.

Conclusions: This study represents the first report C. myriaster from the Zhoushan Fishery being heavily infected with third-stage ascaridoid larvae. Among the ascaridoid larvae parasitic in this fish, an important etiological agent of human anisakidosis, A. pegreffii ( $(\mathrm{3})$, represents the predominant species. The genus Hysterothylacium has the highest species richness, with $H$. fabri (L3) being the most prevalent species. This high level of infection of $A$. pegreffii (L3) in C. myriaster suggests a high risk of anisakidosis or associated allergies for people consuming raw or poorly cooked fish originating from this marine area. These findings provide important basic information on the occurrence and infection parameters of ascaridoid nematodes in this economically important marine fish. They also have significant implications for the prevention and control of human anisakidosis when conger eels from the Zhoushan Fishery are consumed.
\end{abstract}

Keywords: Nematode, Anisakidosis, Ascaridoidea, Conger myriaster, Zhoushan Fishery, East China Sea

\footnotetext{
* Correspondence: liangliangex369@126.com

${ }^{1}$ Key Laboratory of Animal Physiology, Biochemistry and Molecular Biology of

Hebei Province, College of Life Sciences, Hebei Normal University, 050024

Shijiazhuang, Hebei Province, People's Republic of China

Full list of author information is available at the end of the article
}

(c) The Author(s). 2018 Open Access This article is distributed under the terms of the Creative Commons Attribution 4.0 International License (http://creativecommons.org/licenses/by/4.0/), which permits unrestricted use, distribution, and

reproduction in any medium, provided you give appropriate credit to the original author(s) and the source, provide a link to the Creative Commons license, and indicate if changes were made. The Creative Commons Public Domain Dedication waiver (http://creativecommons.org/publicdomain/zero/1.0/) applies to the data made available in this article, unless otherwise stated. 


\section{Background}

Anisakidosis (anisakiasis) is a zoonotic disease wellrecognized by the seafood industry [1-5]. Humans become infected by the accidental ingestion of raw or undercooked fish flesh contaminated by ascaridoid larvae, especially anisakids [5-8]. Most cases of human anisakidosis are caused by Anisakis simplex (sensu stricto), A. pegreffii and Pseudoterranova decipiens, but species of Hysterothylacium and Contracaecum have also been implicated [7, 9-12]. During the past three decades, more than 20,000 cases of human anisakidosis have been reported globally, and over $90 \%$ of cases are from Japan [6]. Although only one case of human anisakidosis has been reported in mainland China [13], due to the spread of exotic foods (sushi, sashimi, etc.) and the growing consumption of raw or undercooked seafood in mainland China, more attention needs to be paid to this disease.

The whitespotted conger Conger myriaster (Brevoort) (Anguilliformes: Congridae) has been considered one of the most common and marketable food-fishes in some Asian countries (i.e. Japan, Korea and China), where it is favoured for consumption raw as sashimi or sushi [1417]. This fish is mainly distributed between the East China Sea and the waters of Korea and Japan [18]. The Zhoushan Fishery off the coast of China is thought to be the most important fishing ground worldwide for $C$. myriaster $[19,20]$. However, there is currently very little information on the levels of infection with ascaridoid parasites in this economically important marine fish. Therefore, the aims of the present study are to determine the species composition, prevalence and mean intensity of ascaridoid parasites in $C$. myriaster caught in the Zhoushan Fishery.

\section{Methods}

Parasite collection

A total of 204 Conger myriaster (Brevoort) (Anguilliformes: Congridae), with a total length (TL) ranging from $25.0-65.0 \mathrm{~cm}$, was dissected, and the body cavity
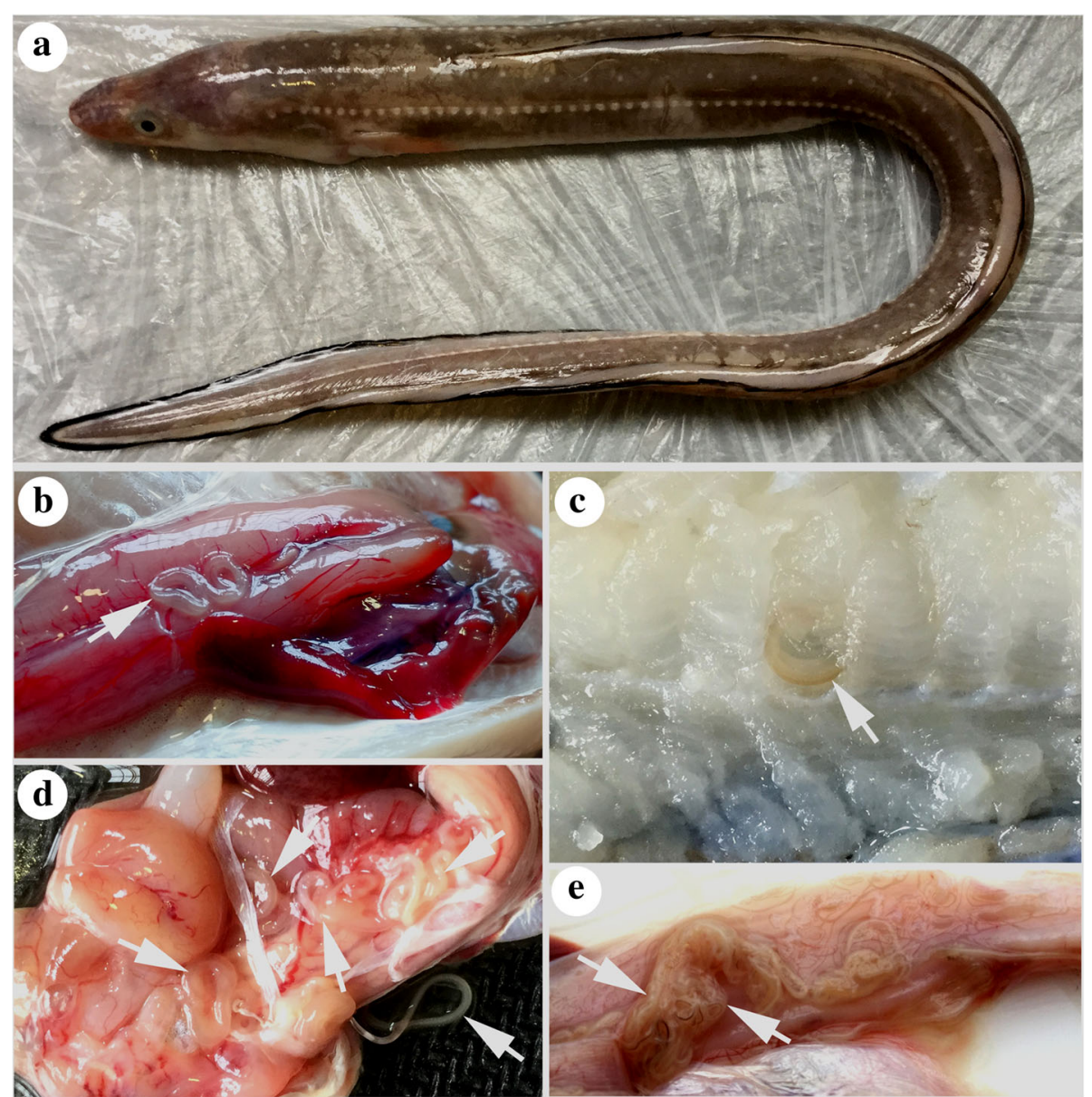

Fig. 1 Conger myriaster (Brevoort) (Anguilliformes: Congridae) caught in the Zhoushan Fishery, China, heavily infected with ascaridoid nematode parasites. a Fish host. $\mathbf{b}, \mathbf{d}$, e Large numbers of ascaridoid nematodes present in the visceral organs. $\mathbf{c}$ Ascaridoid nematode present in the muscles 
and visceral organs (i.e. digestive tract, mesentery, liver and gonads) were examined for nematode parasites (Fig. 1). The musculature of 54 of these fish (26.5\%) (TL 25. $0-50.0 \mathrm{~cm})$ was sliced into thin slivers $(1.0-2.0 \mathrm{~mm}$ thick), and then visually inspected for parasites under white light. All of the fish were caught by commercial trawlers in the Zhoushan Fishery $\left(29^{\circ} 30^{\prime}-31^{\circ} 00^{\prime} \mathrm{N}, 121^{\circ}\right.$ $\left.30^{\prime}-125^{\circ} 00^{\prime} \mathrm{E}\right)$ in the South China Sea off China. The nematodes isolated were washed in physiological saline, then fixed and stored in $80 \%$ ethanol until studied.

\section{Morphological identification}

The morphology of the nematode larvae was observed using light and scanning electron microscopy. For scanning electron microscopy, specimens were prepared according to the methods used in previous studies [21, 22]. The following morphological characters were used for the identification of different morphotypes of larval nematodes as in previous studies [22-27], including the position of the excretory pore, the absence and presence of an intestinal caecum and a ventricular appendix and their relative lengths, and the morphology of the ventriculus and tail tip.

\section{Molecular identification}

For larval morphotypes with large numbers of individuals, the polymerase chain reaction followed by restriction fragment length polymorphism (PCR-RFLP) analysis and targeted sequencing of the internal transcribed spacer (ITS15.8S-ITS2) region of the ribosomal DNA (rDNA) were used for genetic identification. The Column Genomic DNA Isolation Kit (Shanghai Sangon, China) was employed to extract the genomic DNA of each worm according to the manufacturer's instructions. The ITS1-5. 8S-ITS2 region was amplified by PCR using the primers NC5 (5'-GTA GGT GAA CCT GCG GAA GGA TCA T3') and NC2 (5'-TTA GTT TCT TTT CCT CCG CT-3') [28] under the cycling conditions described previously [25]. PCR products were checked on GoldView-stained 1. 5\% agarose gel and purified by the Column PCR Product Purification Kit (Shanghai Sangon, China). PCR-RFLP analysis was performed independently using two restriction enzymes Hinfl and HhaI (Thermo Scientific, Waltham, MA, USA) according to a previous study [29]. The PCR products were digested according to the manufacturer's recommendations. The digested samples were subjected to electrophoresis on $2 \%$ agarose gels and then photographed. Representative samples per distinct RFLP profile set were selected for the sequencing of the ITS1-5. 8S-ITS2 region. Sequencing was carried out using a DyeDeoxyTerminator Cycle Sequencing Kit (v.2, Applied Biosystems, California, USA) and an automated sequencer (ABI-PRISM 377). Sequencing for each sample was carried out for both strands. Sequences were aligned using
ClustalW2 and adjusted manually. The ITS1-5.8S-ITS2 sequences determined were compared (using the algorithm BLASTn) with those available in the National Center for Biotechnology Information (NCBI) database (http://www.ncbi.nlm.nih.gov). For larval morphotypes with small numbers of individuals, the directly targeted sequencing of the ITS1-5.8S-ITS2 region was used for further genetic identification of species with the same primers and methods mentioned above.

\section{Phylogenetic analysis}

Phylogenetic analyses of the ITS1-5.8S-ITS2 sequence data obtained herein were undertaken for both Neighbour-Joining (NJ) and Maximum likelihood (ML) methods using MEGA 6 [30]. We used a built-in function in MEGA 6 [30] to select a best-fitting substitution model for the sequences using the Bayesian information criterion [31]. The Kimura two-parameter model of nucleotide substitution was identified as optimal. Ascaris lumbricoides was chosen as the outgroup. Reliabilities for both NJ and ML trees were tested using 1000 bootstrap replications [32] and nodes with bootstrap values exceeding 70 were considered well supported [33].

\section{Results}

\section{Morphological identification}

A total of 1142 third-stage ascaridoid larvae were isolated from C. myriaster. Based on morphological characters (Figs. 2, 3; Table 1), we found that these larvae represented six different morphotypes belonging to three different genera: Anisakis, Hysterothylacium and Raphidascaris. Among these larvae, 630 individuals were identified morphologically as Anisakis type I of Berland (1961) [23]. Four different morphotypes of Hysterothylacium spp. larvae were distinguished, including the Hysterothylacium larval type of Smith (1983) [24] with 28 individuals, Hysterothylacium larval type of Guo et al. (2014) [27] with 23 individuals, Hysterothylacium larval type of Li et al. (2012) [25] with 11 individuals and Hysterothylacium larval type IV of Shamsi et al. (2013) [26] with 447 individuals. Only three third-stage larvae of Raphidascaris, identified morphologically as Raphidascaris larval type of Zhao et al. (2016) [22], were found in the present study (see Table 2 for details).

\section{Molecular identification}

All the Anisakis samples were further identified by PCRRFLP analysis (Table 2). Digestion of the PCR products using HhaI produced two different RFLP profiles, i.e. 628 samples with two bands (c.550 and $430 \mathrm{bp}$ ) and only two samples with four bands (c.320, 240, 180 and 160 bp). Digestion with Hinfl yielded three different RFLP profiles, 621 samples with three bands (c.370, 330 and $250 \mathrm{bp}$ ), only two samples with two bands (c.620 and 


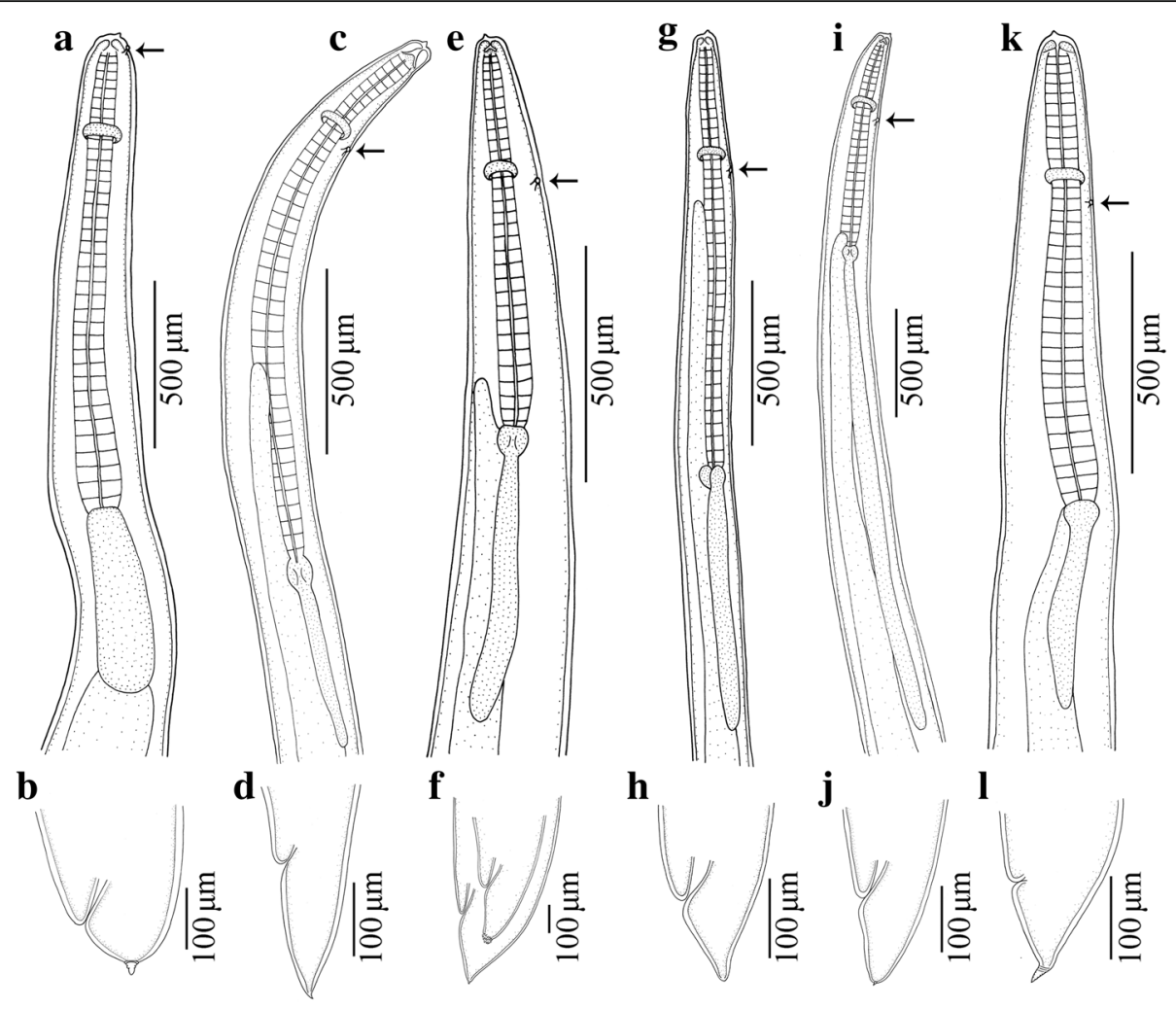

Fig. 2 Anterior and posterior extremities of ascaridoid larval morphotypes isolated from Conger myriaster (Brevoort) (Anguilliformes: Congridae) caught in the Zhoushan Fishery, China (excretory pore arrowed). a, b, Anisakis type I of Berland (1961) [23]. c, d, Hysterothylacium larval type of Smith (1983) [24]. e, f Hysterothylacium larval type IV of Shamsi et al. (2013) [26]. g, h Hysterothylacium larval type of Guo et al. (2014) [27]. i, $\mathbf{j}$ Hysterothylacium larval type of Li et al. (2012) [25]. k, I Raphidascaris larval type of Zhao et al. (2016) [22]

$350 \mathrm{bp})$ and seven samples with four bands $(c .620,370$, 300 and $250 \mathrm{bp})$. According to the molecular taxonomic key based on the PCR-RFLP patterns of Anisakis species obtained by the digestion of ITS amplicons with endonucleases HhaI or Hinfl [29], we assigned these Anisakis third-stage larvae to A. pegreffii, A. typica and A. simplex (sensu stricto) $\times A$. pegreffii (a recombinant genotype). The ITS region was sequenced for 43 randomly selected individuals of $A$. pegreffii, two individuals of $A$. typica and seven individuals of $A$. simplex (sensu stricto) $\times A$. pegreffii identified by the PCR-RFLP analysis (Table 2). There was no nucleotide variation detected among the 43 ITS sequences of $A$. pegreffii (MF539758-MF539767), two ITS sequences of A. typica (MF539771, MF539775) and seven ITS sequences of $A$. simplex (sensu stricto) $\times$ A. pegreffii (MF539768-MF539770, MF539772MF539774, MF539776). Pairwise comparison between our genetic data and the ITS sequences of Anisakis spp. registered in GenBank proved $100 \%$ identical to $A$. pegreffii (AY821738, AY821740, AY821745, JQ934867, JQ934871, JQ900763, JQ934869, KP301519, KF032066, KJ011486, EU624343), A. typica (EU346093, KC928262, KF356670-KF356671, JX523715, JN968930) and A. simplex (sensu stricto $) \times$ A. pegreffii $(\mathrm{AB} 894874)$.
For the further identification of Hysterothylacium spp. third-stage larvae, the ITS region was sequenced for 45 randomly selected individuals of Hysterothylacium larval type IV of Shamsi et al. (2013) [26] and all of the individuals of the other three larval morphotypes (Table 2). Four different genotypes (MF539793, MF539794, MF539796, MF539790) were detected among the 45 ITS sequences of Hysterothylacium larval type IV of Shamsi et al. (2013) [26] (MF539787-MF539796) obtained herein, which displayed $0-0.2 \%$ nucleotide variability. Pairwise comparisons between the present data and the ITS sequences of Hysterothylacium spp. registered in GenBank showed 0-0. 3\% nucleotide differences with $H$. fabri (KC852206, JQ520158, JX974558, KF736939-KF736944). Thus, we considered that the present nematode larvae, referred to as Hysterothylacium larval type IV of Shamsi et al. (2013) [26], belong to $H$. fabri. No nucleotide variability was detected among the 23 ITS sequences of Hysterothylacium larval type HL of Guo et al. (2014) [27] (MF539797MF539806). Pairwise comparisons between the present data and the ITS sequences of Hysterothylacium spp. registered in GenBank showed them to be $100 \%$ identical with H. sinense (KX817293-KX817295, KX110078, KX084795). Thus, we confirmed that the nematode larvae 

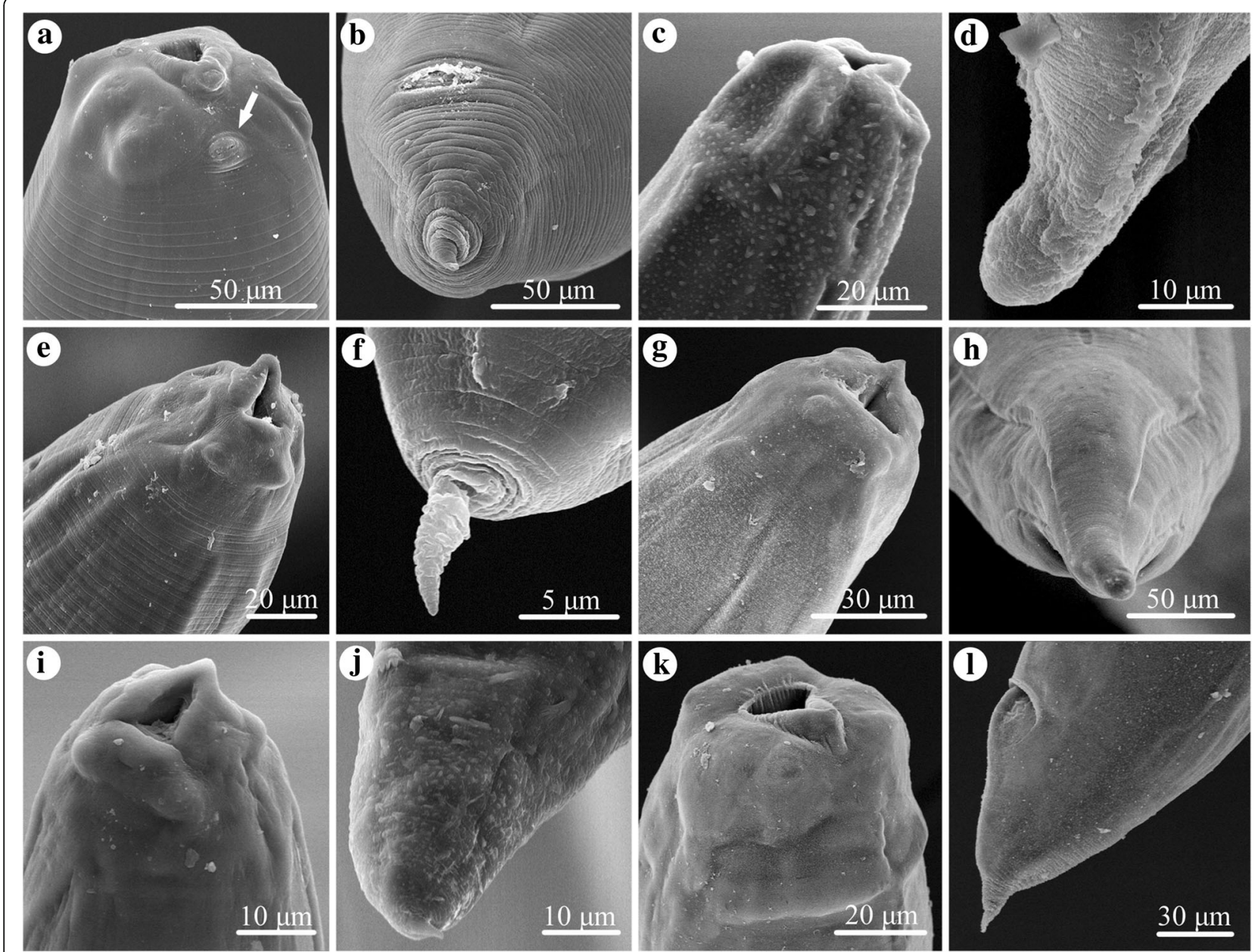

Fig. 3 Scanning electron micrographs of the cephalic extremity and tail of ascaridoid larval morphotypes isolated from Conger myriaster (Brevoort) (Anguilliformes: Congridae) caught in the Zhoushan Fishery, China. a, b Anisakis type I of Berland (1961) [23] (excretory pore arrowed). c, d Hysterothylacium larval type of Guo et al. (2014) [27]. e, f Hysterothylacium larval type of Smith (1983) [24]. g, h Hysterothylacium larval type of Li et al. (2012) [25]. i, j Hysterothylacium larval type IV of Shamsi et al. (2013) [26]. k, I Raphidascaris larval type of Zhao et al. (2016) [22]

referred to as Hysterothylacium larval type HL of Guo et al. (2014) [27] belong to $H$. sinense. Only one genotype was detected among the 28 ITS sequences of specimens referred to as Hysterothylacium larval type of Smith (1983) [24] (MF539777-MF539786). Pairwise comparisons between the present data and the ITS sequences of Hysterothylacium spp. registered in GenBank showed 0-0.5\% nucleotide differences with $H$. aduncum (KF736937, HM437225, KP318743-КР318782, KP276150, КT852542). Thus, we considered that the specimens referred to as Hysterothylacium larval type of Smith (1983) [24] represent $H$. aduncum. There were seven different genotypes detected among the 11 ITS sequences of Hysterothylacium larval type of Li et al. (2012) [25] (MF539807-MF539813, MF539814-MF539817) obtained herein, which displayed $0-1.7 \%$ nucleotide variability (Table 3 ). Pairwise comparisons between the present data and the ITS sequences of
Hysterothylacium spp. registered in GenBank showed that seven individuals (MF539807-MF539813) exhibited 0-0. 3\% nucleotide differences with $H$. amoyense (KP252130KP252133, EU828749), and four individuals (MF539814MF539817) exhibited 0-1.4\% nucleotide differences with H. zhoushanense (KP326556, KP326549-KP326551, JX028277-JX028282). We considered, therefore, that the 11 individuals of Hysterothylacium larval type of $\mathrm{Li}$ et al. (2012) [25] represent two different species, H. zhoushanense and $H$. amoyense, with $0.8-1.7 \%$ nucleotide variation in the ITS region between these species. Only three thirdstage larvae of Raphidascaris, identified morphologically as Raphidascaris larval type of Zhao et al. (2016) [22], were found. The ITS region was directly sequenced for all of these Raphidascaris larvae (Table 2) and the three ITS sequences (MF539818-MF539820) were identical. Comparison of our genetic data with the ITS sequences of 
Table 1 Morphometric data for ascaridoid larval morphotypes isolated from Conger myriaster (Brevoort) (Anguilliformes: Congridae) caught in the Zhoushan Fishery, China (measurements in mm)

\begin{tabular}{lllllll}
\hline & $\begin{array}{l}\text { ATB } \\
(n=10)\end{array}$ & $\begin{array}{l}\text { HTS } \\
(n=10)\end{array}$ & $\begin{array}{l}\text { HTIV } \\
(n=10)\end{array}$ & $\begin{array}{l}\text { HTG } \\
(n=10)\end{array}$ & $\begin{array}{l}\text { HTL } \\
(n=8)\end{array}$ & $\begin{array}{l}\text { RTZ } \\
(n=3)\end{array}$ \\
\hline BL & $9.0-25.0$ & $8.0-21.0$ & $7.0-20.0$ & $9.0-23.0$ & $6.0-19.0$ & $7.0-8.0$ \\
OL & $0.84-1.93$ & $1.12-1.85$ & $0.62-1.31$ & $1.15-2.18$ & $0.63-1.31$ & $0.66-0.70$ \\
VL & $0.40-0.79$ & $0.07-0.10$ & $0.05-0.15$ & $0.05-0.10$ & $0.05-0.15$ & 0.05 \\
WW & $0.11-0.30$ & $0.06-0.10$ & $0.05-0.14$ & $0.05-0.09$ & $0.05-0.15$ & $0.08-0.09$ \\
ICL & - & $0.46-0.74$ & $0.13-0.39$ & $0.67-1.29$ & $0.09-0.18$ & - \\
VAL & - & $0.42-1.27$ & $0.78-2.43$ & $0.70-1.39$ & $1.26-6.29$ & $0.37-0.44$ \\
EC & at base of lip & $0.35-0.48$ & $0.27-0.47$ & $0.35-0.57$ & $0.28-0.48$ & $0.26-0.27$ \\
TL & $0.059-0.15$ & $0.14-0.27$ & $0.10-0.27$ & $0.09-0.19$ & $0.12-0.23$ & $0.12-0.13$ \\
OL/BL (\%) & $7.5-13.1$ & $8.8-14.0$ & $6.2-8.9$ & $9.5-13.5$ & $5.2-11.3$ & $8.8-9.4$ \\
ICL:VAL ratio & - & $1: 0.8-2.2$ & $1: 3.2-8.1$ & $1: 0.9-1.4$ & $1: 8.7-48.8$ & - \\
ICL/OL (\%) & - & $24.7-45.2$ & $19.2-37.3$ & $58.1-71.1$ & $10.2-15.8$ & - \\
\hline
\end{tabular}

Abbreviations: $B L$ body length, $O L$ oesophagus length, $V L$ ventriculus length, $V W$ ventriculus width, $I C L$ intestinal caecum length, $V A L$ ventricular appendix length, EC distance from excretory pore to cephalic end, TL tail length, ATB Anisakis type I of Berland (1961) [23], HTS Hysterothylacium larval type of Smith (1983) [24], HTG Hysterothylacium larval type of Guo et al. (2014) [27], HTL Hysterothylacium larval type of Li et al. (2012) [25], HTIV Hysterothylacium larval type IV of Shamsi et al. (2013) [26], RTZ Raphidascaris larval type of Zhao et al. (2016) [22]

Raphidascaris spp. registered in GenBank showed them to be 100\% identical with $R$. lophii (KP262039, KP326520KP326531, KP326533-KP326538, KP419720). Consequently, we considered these Raphidascaris larvae to be conspecific with $R$. lophii. All of the ITS sequences of the larval nematode parasites obtained herein are deposited in the GenBank database under the accession numbers (MF539758-MF539820).

\section{Phylogenetic analysis}

Our results revealed that the ascaridoid nematodes selected for phylogenetic analysis were divided into two distinct clades (families), the Anisakidae (which includes species of Anisakis, Pseudoterranova and Contracaecum) and the Raphidascarididae (which includes species of Hysterothylacium and Raphidascaris) with strong support (Fig. 4). In Anisakis, A. simplex (JX535521), A.

Table 2 Infection data of ascaridoid nematode larvae isolated from Conger myriaster (Brevoort) (Anguilliformes: Congridae) caught in the Zhoushan Fishery, China, and samples selected for molecular analysis

\begin{tabular}{|c|c|c|c|c|c|c|c|}
\hline Morphotype & Species & $\begin{array}{l}\text { Prevalence } \\
(\%)\end{array}$ & $\begin{array}{l}\text { Intensity } \\
\text { range } \\
\text { (mean) }\end{array}$ & $\begin{array}{l}\text { No. of specimens } \\
\text { isolated from fish }\end{array}$ & $\begin{array}{l}\text { No. of specimens } \\
\text { used for PCR-RFLP } \\
\text { analysis }\end{array}$ & $\begin{array}{l}\text { No. of specimens } \\
\text { used for } \\
\text { sequencing }\end{array}$ & GenBank ID \\
\hline$\overline{\text { ATB }}$ & Anisakis pegreffii & 99.0 & $1-15(3.1)$ & 621 & 621 & $43^{a}$ & MF539758-MF539767 \\
\hline ATB & $\begin{array}{l}\text { Anisakis pegreffii } \\
\times \text { A. simplex }\end{array}$ & 3.4 & $1(1.0)$ & 7 & 7 & 7 & $\begin{array}{l}\text { MF539768-MF539770; } \\
\text { MF539772-MF539774; } \\
\text { MF539776 }\end{array}$ \\
\hline ATB & Anisakis typica & 1.0 & $1(1.0)$ & 2 & 2 & 2 & MF539771, MF539775 \\
\hline HTIV & $\begin{array}{l}\text { Hysterothylacium } \\
\text { fabri }\end{array}$ & 47.1 & $1-41(4.7)$ & 447 & 447 & $45^{\mathrm{a}}$ & MF539787-MF539796 \\
\hline HTS & $\begin{array}{l}\text { Hysterothylacium } \\
\text { aduncum }\end{array}$ & 11.8 & $1-5(1.2)$ & 28 & 0 & 28 & MF539777-MF539786 \\
\hline HTL & $\begin{array}{l}\text { Hysterothylacium } \\
\text { amoyense }\end{array}$ & 3.4 & $1-2(1.2)$ & 7 & 0 & 7 & MF539807-MF539813 \\
\hline HTL & $\begin{array}{l}\text { Hysterothylacium } \\
\text { zhoushanense }\end{array}$ & 2.0 & $1(1.0)$ & 4 & 0 & 4 & MF539814-MF539817 \\
\hline HTG & $\begin{array}{l}\text { Hysterothylacium } \\
\text { sinense }\end{array}$ & 10.3 & $1-2(1.1)$ & 23 & 0 & 23 & MF539797-MF539806 \\
\hline RTZ & $\begin{array}{l}\text { Raphidascaris } \\
\text { lophii }\end{array}$ & 1.5 & $1(1.0)$ & 3 & 0 & 3 & MF539818-MF539820 \\
\hline
\end{tabular}

${ }^{\text {a Randomly selected }}$

Abbreviations: ATB Anisakis type I of Berland (1961) [23], HTS Hysterothylacium larval type of Smith (1983) [24], HTG Hysterothylacium larval type of Guo et al. (2014) [27], HTL Hysterothylacium larval type of Li et al. (2012) [25], HTIV Hysterothylacium larval type IV of Shamsi et al. (2013) [26], RTZ Raphidascaris larval type of Zhao et al. (2016) [22] 
Table 3 Sequence polymorphisms (highlighted in bold) revealed at alignment positions of the ITS region among the different individuals of Hysterothylacium larval type of Li et al. (2012) [25] obtained in the present study

\begin{tabular}{|c|c|c|c|c|c|c|c|c|c|c|c|c|c|c|c|c|c|c|}
\hline & \multicolumn{18}{|c|}{ Sequence polymorphisms at alignment positions } \\
\hline & 96 & 97 & 98 & 220 & 342 & 392 & 685 & 690 & 691 & 696 & 698 & 710 & 807 & 808 & 809 & 812 & 823 & 861 \\
\hline \multicolumn{19}{|l|}{ H. amoyense } \\
\hline MF539809 & - & - & - & $\mathrm{T}$ & T & A & C & A & A & A & A & C & - & - & - & C & G & A \\
\hline MF539811 & - & - & - & $\mathrm{T}$ & T & A & C & A & C & A & A & C & - & - & - & C & G & A \\
\hline MF539810 & - & - & - & $\mathrm{T}$ & $\mathrm{T}$ & A & C & A & C & A & A & C & - & - & - & C & G & A \\
\hline MF539813 & - & - & - & $\mathrm{T}$ & T & A & C & A & C & A & A & C & - & - & - & C & G & A \\
\hline MF539808 & - & - & - & $\mathrm{T}$ & T & A & C & A & C & A & G & C & - & - & - & C & G & A \\
\hline MF539807 & - & - & - & $\mathrm{T}$ & T & A & C & A & C & A & G & C & - & - & - & C & G & A \\
\hline MF539812 & - & - & - & $\mathrm{T}$ & T & A & C & A & C & A & G & C & - & - & - & C & G & A \\
\hline \multicolumn{19}{|c|}{ H. zhoushanense } \\
\hline MF539814 & $\mathbf{T}$ & G & $\mathbf{G}$ & $\mathrm{T}$ & A & G & C & $\mathbf{T}$ & C & G & A & G & $T$ & $\mathbf{T}$ & G & G & $\mathbf{T}$ & G \\
\hline MF539816 & - & - & - & $\mathrm{C}$ & A & G & C & $\mathrm{T}$ & C & G & A & G & $T$ & $\mathbf{T}$ & G & G & $\mathbf{T}$ & G \\
\hline MF539817 & - & - & - & $\mathrm{C}$ & A & A & G & $T$ & C & G & A & G & - & - & - & G & $\mathbf{T}$ & G \\
\hline MF539815 & - & - & - & $\mathrm{T}$ & T & A & G & $T$ & C & G & A & G & - & - & - & G & $\mathbf{T}$ & G \\
\hline
\end{tabular}

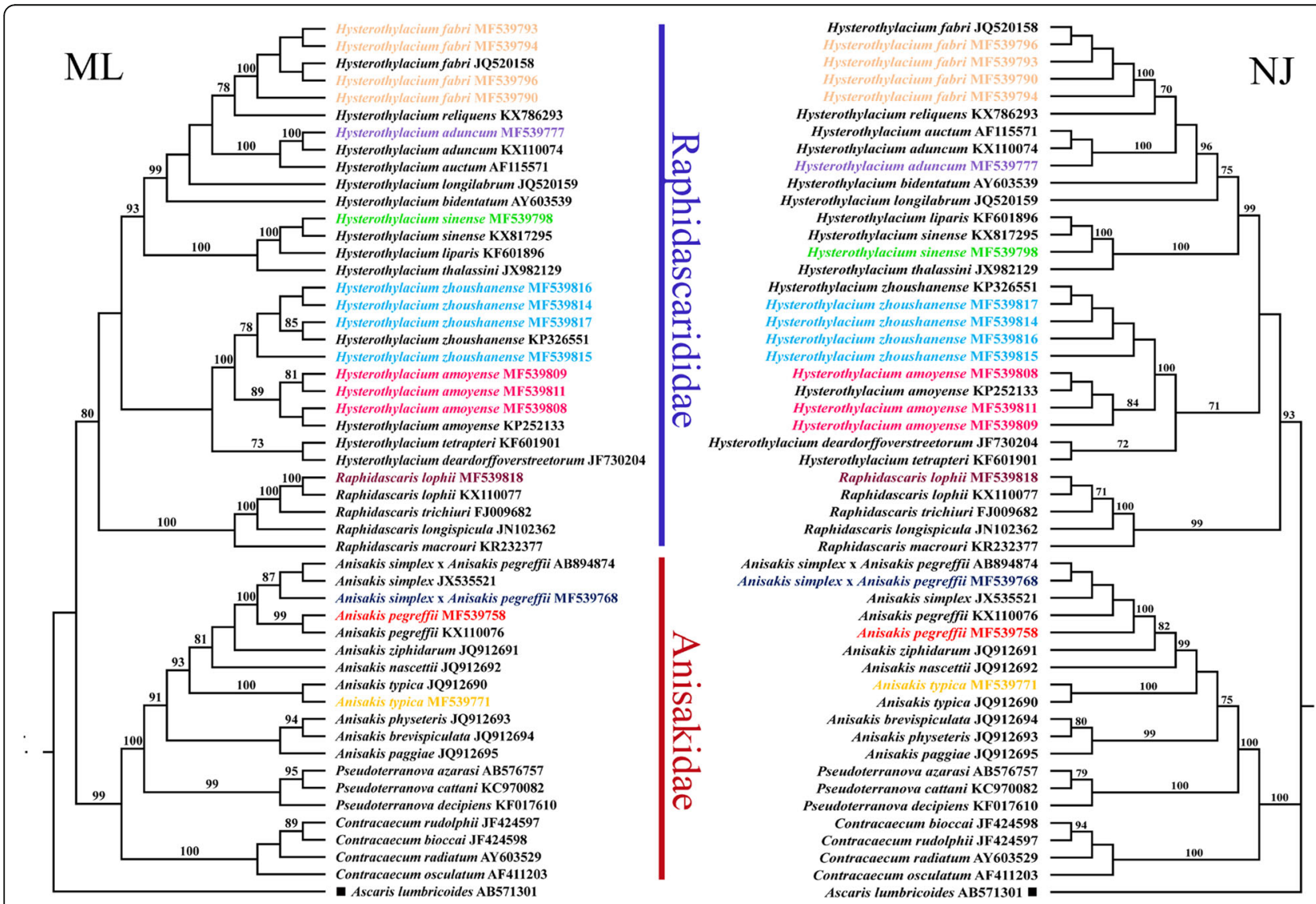

Fig. 4 Maximum likelihood (ML) and Neighbour-Joining (NJ) trees showing the phylogenetic relationships of ascaridoid nematode species detected in the present study (shown in colour). Ascaris lumbricoides was chosen as the outgroup 
pegreffii (MF539758, KX110076), A. simplex (sensu stricto) $\times$ A. pegreffii (MF539768, AB894874), A. ziphidarum (JQ912691), A. nascettii (JQ912692) and A. typica (MF539771, JQ912690) grouped together, representing Anisakis type I of Berland (1961) [23], and A. brevispiculata (JQ912694), A. paggiae (JQ912695) and A. physeteris (JQ912693) formed a group, representing Anisakis type II. The results showed that $A$. pegreffii, $A$. simplex and $A$. simplex (sensu stricto) $\times A$. pegreffii exhibit a very close relationship and that $A$. typica was the basal branch in the Anisakis type I group. In Hysterothylacium, the four different genotypes of $H$. fabri (MF539793, MF539794, MF539796, MF539790) obtained herein and the previously reported ITS sequence of $H$. fabri (JQ520158) clustered together and as a sistergroup to $H$. reliquens (KX786293). Hysterothylacium sinense (MF539798, KX817295) formed a sister relationship with H. liparis (KF601896). Hysterothylacium aduncum (MF539777, KX110074) and H. auctum (AF115571) also exhibited a close relationship (Fig. 4). The seven different genotypes of Hysterothylacium larval type of Li et al. (2012) [25] obtained herein were divided into two clades: three genotypes (MF539808, MF539809, MF539811) clustered with the previously reported ITS data for H. amoyense (KP252133) and four genotypes (MF539814-MF539817) grouped together with a previously reported ITS sequence of $H$. zhoushanense (KP326551) (Fig. 4). Raphidascaris lophii (MF539818) was sister to $R$. trichiuri (FJ009682) with high branch support scores (Fig. 4).

\section{Infection levels}

The overall prevalence of infection for ascaridoid larvae in C. myriaster was $100 \%$ (mean intensity 5.6 worms/ fish). Anisakis pegreffii (L3) was the predominant species. The prevalence and mean intensity of $A$. typica and A. simplex (sensu stricto) $\times$ A. pegreffii was distinctly lower than that of $A$. pegreffii. Hysterothylacium fabri (L3) represented the most prevalent species among the Hysterothylacium larvae collected from C. myriaster. The infection parameters of the other four species of Hysterothylacium and R. lophii are presented in Table 2. Moreover, in the 54 fish whose muscles were sliced into thin slivers, only seven Anisakis larvae, identified genetically as $A$. pegreffii, were found in the musculature, with a prevalence of $2.5 \%$ and a mean intensity of 1.0 .

\section{Discussion}

Conger myriaster represents one of the most valuable fishery resources in some Asian countries (i.e. Japan, Korea and China) [14-17], but it has also been considered an extremely important agent of anisakidosis in Korea [15, 34]. However, our present knowledge of the species composition, prevalence and mean intensity of ascaridoid parasites in C. myriaster remains very limited. To date, only one preliminary investigation of the occurrence of ascaridoid larvae in this fish has been undertaken; it was carried out in 1992 and based on specimens purchased from a fish market in Seoul [34]. The study showed that the overall prevalence of infection was $57.7 \%$ (of the only 26 fish investigated) [34] and distinctly lower than that (100\%) found in C. myriaster collected from the Zhoushan Fishery in Chinese waters. However, the mean intensity (90.1) in the previous study is much higher than the value in our investigation (mean intensity only 5.6). The different infection parameters of ascaridoid larvae in C. myriaster caught in Korean and Chinese waters may be a result of the different geographical locations or the sampling time (July to August in 1990 vs October in 2010 and 2016 and April in 2012 and 2013). In addition, the species composition of ascaridoid larvae detected from C. myriaster caught in Korean and Chinese waters is similar. These larval types, i. e. Anisakis type I of Berland (1961) [23], Hysterothylacium larval type of Smith (1983) [24], Hysterothylacium larval type IV of Shamsi et al. (2013) [26] and Hysterothylacium larval type of Guo et al. (2014) [27], were found in both our study and the previous one [34], but Raphidascaris larval type of Zhao et al. (2016) [22] is only reported in our study. Unfortunately, in the previous study these larvae were not accurately determined to the species level because only morphological methods were used.

The accurate identification of ascaridoid larvae to the species level is essential for an evaluation of the molecular epidemiology of the disease [21, 22, 35]. Recently, the combination of PCR-RFLP analysis and targeted sequencing of the ITS region has been widely used for largescale studies on the identification of ascaridoid larvae to the species level $[11,29,35-40]$. Consequently, in the present study, in order to accurately identify large numbers of ascaridoid larvae isolated from C. myriaster in the Zhoushan Fishery, both morphological methods and molecular approaches, including PCR-RFLP analysis and/or targeted sequencing of the ITS region, were employed, and the six ascaridoid larval types classified herein were identified genetically as nine species.

According to some previous studies [12, 41-45], most cases of human anisakidosis in Europe and Korea are known to be caused by $A$. pegreffii (L3), which is widely distributed in the South Atlantic, the Mediterranean Sea, Australian and Chinese waters (including the Yellow Sea, the East China Sea and the Taiwan Strait) [27, 29, 35, 36, 40, 46-50]. During the present survey, a high level of $A$. pegreffii infection with an overall prevalence of $99.0 \%$, was also revealed in C. myriaster, but most of the $A$. pegreffii were found in the body cavity and visceral organs of the fish, which are not eaten by humans, 
and only seven third-stage larvae of $A$. pegreffii were detected from the musculature of seven $C$. myriaster (prevalence $13.0 \%$ and mean intensity 1.0 ). The relatively low prevalence and intensity of Anisakis specimens detected in the musculature of $C$. myriaster suggest a relatively low probability of human infection when consuming C. myriaster as sashimi or sushi. However, the post-mortem migration of Anisakis larvae from the fish body cavity and/or visceral organs to the musculature can occur after the death of the fish [7, 51, 52], which could increase the risk of anisakidosis when consuming raw or undercooked fish. Consequently, we suggest the removal of the viscera from $C$. myriaster as soon after capture as possible, which would be a useful and practical preventive measure against human anisakidosis [15].

Our genetic data and phylogenetic analysis indicated that the third-stage larvae of Anisakis type I of Berland (1961) [23] obtained herein represent three species: $A$. pegreffii, A. typica and A. simplex (sensu stricto) $\times A$. pegreffii (Fig. 4). However, based only on morphological characters (i.e. the morphology of the cephalic region, the length of the oesophagus, and the length and morphology of the ventriculus and tail), it is almost impossible to distinguish the third-stage larvae of the three species [37, 46]. The infection levels of A. typica (L3) and A. simplex (sensu stricto) $\times A$. pegreffii (L3) are distinctly lower than that of A. pegreffii (see Table 2 for details). This situation can be readily understood if one considers the different distributional patterns of these Anisakis species in Chinese waters. Anisakis typica is mainly distributed in the tropical and subtropical waters [48] and is considered to be the predominant species in the South China Sea [37], but it has also been reported from marine fish species in other Chinese waters (i.e. Auxis tapeinosoma and Chelidonichthys kumu in the Yellow Sea, Trichiurus lepturus in the East China Sea and Scomber australasicus in the Taiwan Strait) $[29,40,46]$. The recombinant genotype $A$. simplex (sensu stricto) $\times A$. pegreffii is found in both temperate and subtropical waters and has frequently been reported from various marine fishes in the Yellow Sea, East China Sea and Taiwan Strait, but all reports indicate low levels of prevalence and intensity $[40,46,53]$. The present study is the first record of $A$. simplex (sensu stricto) $\times$ A. pegreffii from C. myriaster in the East China Sea. The significance of $A$. typica and A. simplex (sensu stricto) $\times$ A. pegreffii as causative agents of human anisakidosis is distinctly lower than that of $A$. pegreffii. To date, only one case of human anisakidosis caused by the recombinant genotype $A$. simplex (sensu stricto) $\times A$. pegreffii has been reported; this was from Japan [54]. Anisakis typica has not been confirmed as a pathogen causing human anisakidosis.

Species of Hysterothylacium are common nematode parasites of marine fishes worldwide [27, 35]. Marine fishes can act as both the paratenic/intermediate and/or the definitive hosts of Hysterothylacium spp. [55, 56]. However, most of species of this genus are commonly considered as non-pathogenic to humans. So far, only one case of human anisakidosis apparently caused by $H$. aduncum has been reported [9]. Among the ascaridoid larvae parasitic in C. myriaster, Hysterothylacium has the highest species richness (five species). Based on the relative length of the intestinal caecum and ventricular appendix, and the morphology of the tail, Hysterothylacium larvae can be readily assigned to four different morphotypes (Figs. 2, 3, Table 1). However, it is impractical and problematic to identify these different larval morphotypes to the species level using morphological characters alone [25], thus molecular data were used for the exact identification of species. Hysterothylacium fabri (L3) was the most prevalent species among the Hysterothylacium spp. larvae obtained herein. The prevalence of $H$. fabri (L3) in C. myriaster was similar to that reported for Liparis tanakae (Gilbert \& Burke) (Scorpaeniformes: Liparidae) collected from the Yellow and East China Seas (prevalence 30.0\%) [27]. The present study represents the first record of $\mathrm{H}$. fabri (L3) in C. myriaster. Hysterothylacium aduncum is the most common marine ascaridoid parasite, being reported from more than 220 fish species belonging to 70 families in 22 orders throughout the world [57]. Recently, some authors reported H. aduncum (L3) in L. tanakae (prevalence of $100 \%$, mean intensity 26.7) and Pseudorhombus cinnamoneus (Temminck \& Schlegel) (Pleuronectiformes: Paralichthyidae) (prevalence 81.2\%, mean intensity 2.7) in Chinese waters [27, 35]. The prevalence and mean intensity of $H$. aduncum (L3) in these two studies were considerably higher than the values in the present study (prevalence $11.8 \%$, mean intensity 1.2). Hysterothylacium sinense possibly represents a species endemic to Chinese waters; its type-host is $C$. myriaster. The thirdstage larvae of $H$. sinense have been reported from $P$. cinnamoneus in the Yellow Sea off China with a prevalence of $100 \%$ and mean intensity of 17.4 [35]. In contrast, we found $H$. sinense (L3) from C. myriaster in the East China Sea with a prevalence of only $10.3 \%$ and a mean intensity of 1.1. Hysterothylacium amoyense (L3) has been reported from several marine fishes, including Scomber japonicus and Trichiurus lepturus in the East China Sea, Halieutaea stellata in the South China Sea [21, 39] and Platycephalus indicus (L.) (Scorpaeniformes: Platycephalidae) in the Persian Gulf [58]. In the present study, the prevalence of $H$. amoyense (L3) (3.4\%) in $C$. myriaster is distinctly lower than reported in the previous investigation of Halieutaea stellata (24.0\%) [21]. In the case of the third-stage larvae of $H$. zhoushanense, we found this species in C. myriaster for the first time, with a prevalence of $2.0 \%$ and mean intensity of 1.0. Previously, 
it has been reported from Pseudorhombus oligodon (Bleeker) (Pleuronectiformes: Paralichthyidae) in the East China Sea, with a prevalence of $5.3 \%$ and mean intensity of 7.0 [25].

Our phylogenetic analyses showed that $A$. pegreffii, $A$. simplex (sensu stricto) $\times A$. pegreffii and $A$. simplex (sensu stricto) have much closer relationships than with A. typica. Consequently, the present results agree with several previous phylogenetic studies [22, 35, 37, 40, 47, 48]. Some authors have considered that $H$. zhoushanense is closely related to $H$. amoyense, and it is indeed almost impossible to distinguish the third-stage larvae of these two species based on morphological characters [25]. However, adults of $H$. zhoushanense, which have welldeveloped lateral alae, can be readily differentiated from those of $H$. amoyense, which lack alae. Our phylogenetic analysis corroborated $H$. zhoushanense and $H$. amoyense as separate species and confirmed their sister relationship (Fig. 4).

Conger myriaster is bathydemersal and a voracious predator. The high levels of nematode infections and species richness in this fish host are likely related to its feeding habit, which is the main factor affecting parasite community structure [59]. To date, more than 50 species of marine fishes, 40 species of crustaceans, 15 species of molluscs and four species of annelids have been reported in its diet [60]. Most of these prey fishes and invertebrates can act as paratenic or intermediate hosts of the above-mentioned ascaridoid nematode species. We strongly recommended two traditional methods for reducing the risk of human anisakidosis when C. myriaster from the Zhoushan Fishery is consumed, i.e. adequate cooking $\left(60{ }^{\circ} \mathrm{C}\right.$ for $\left.1-2 \mathrm{~min}\right)$ or freezing $\left(-10{ }^{\circ} \mathrm{C}\right.$ for $\left.24 \mathrm{~h}\right)$ of the fish, both of which should kill anisakids [61]. Lastly, the visual inspection of pieces of fish for parasites when preparing sashimi or sushi may be also a good prophylactic measure against human anisakidosis, because Anisakis larvae can be detected and eliminated if the pieces of fish are sliced thinly.

\section{Conclusions}

Conger myriaster caught in the Zhoushan Fishery was heavily infected with ascaridoid third-stage larvae. Among the ascaridoid larvae parasitic in this fish, the important etiological agent of human anisakidosis, $A$. pegreffii (L3), represented the predominant species. This high infection level of $A$. pegreffii (L3) in C. myriaster suggests a high risk of anisakidosis or associated allergies for people consuming raw or poorly cooked raw fish originating from this marine area. The genus Hysterothylacium had the highest species richness, and H. fabri (L3) was the most prevalent species among the Hysterothylacium larvae. The findings of the present study provide important basic data on the occurrence and infection parameters of ascaridoid nematodes in this economically important marine fish. They also have significant implications for the prevention and control of human anisakidosis, which is possible when the flesh of fish caught in the Zhoushan Fishery off the coast of China is consumed raw or inadequately cooked.

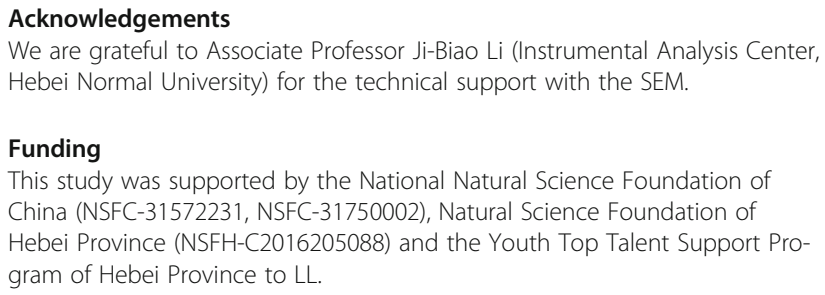

\section{Availability of data and materials}

ITS sequences of the ascaridoid nematode parasites obtained in this study were deposited in the GenBank database under the accession numbers MF539758-MF539820. Voucher specimens are deposited in College of Life Sciences, Hebei Normal University, Hebei Province, China, under the accession numbers HBNU-F2017020L-F2017029L.

\section{Authors' contributions}

HXC, LPZ and LL conceived the study, identified and sequenced samples, and analysed the data. HXC, ZX, HTL, HDJ and LL collected the parasites from fish. LLü contributed to the phylogeny. LL and DIG wrote the manuscript. All authors read and approved the final manuscript.

\section{Ethics approval}

This study was conducted according to the Hebei Normal University Experiments in Animals Policy and was approved by the Animal Ethics Committee of Hebei Normal University as complying with the Animal Protection Law of the People's Republic of China.

\section{Competing interests}

The authors declare that they have no competing interests.

\section{Publisher's Note}

Springer Nature remains neutral with regard to jurisdictional claims in published maps and institutional affiliations.

\section{Author details}

${ }^{1}$ Key Laboratory of Animal Physiology, Biochemistry and Molecular Biology of Hebei Province, College of Life Sciences, Hebei Normal University, 050024

Shijiazhuang, Hebei Province, People's Republic of China. ${ }^{2}$ Department of Life Sciences, Natural History Museum, Cromwell Road, London SW7 5BD, UK.

${ }^{3}$ Medical College of Hebei University of Engineering, 056002 Handan, Hebei Province, People's Republic of China.

Received: 28 December 2017 Accepted: 16 April 2018

Published online: 02 May 2018

\section{References}

1. Ahmed FE. Review: Assessing and managing risk due to consumption of seafood contaminated with micro-organisms, parasites, and natural toxins in the US. Int J Food Sci Tech. 1992;27:243-60.

2. Butt AA, Aldridge $K E$, Sanders $C V$. Infections related to the ingestion of seafood. Part I: Parasitic infections and food safety. Lancet Infect Dis. 2004;4: 294-300.

3. Chai JY, Murrell KD, Lymbery AJ. Fish-borne parasitic zoonoses: status and issues. Int J Parasitol. 2005:35:1233-54

4. Anantanawat S, Kiermeier A, McLeod C, Sumner J. A semi-quantitative risk assessment of harmful parasites in Australian finfish. South Australia: South Australian Research \& Development Institute; 2012. p. 36.

5. Audicana MT, Ansotegui IJ, de Corres LF, Kennedy MW. Anisakis simplex: dangerous - dead and alive? Trends Parasitol. 2002;18:20-5. 
6. Hochberg NS, Hamer DH. Anisakidosis: perils of the deep. Clin Infect Dis. 2010;51:806-12.

7. Suzuki J, Murata R, Hosaka M, Araki J. Risk factors for human Anisakis infection and association between the geographic origins of Scomber japonicus and anisakid nematodes. Int J Food Microbiol. 2010;31:88-93.

8. Shamsi S, Suthar J. A revised method of examining fish for infection with zoonotic nematode larvae. Int J Food Microbiol. 2016;227:13-6.

9. Yagi K, Nagasawa K, Ishikura H, Nakagawa A, Sato N, Kikuchi K, et al. Female worm Hysterothylacium aduncum excreted from human: a case report. Jpn J Parasitol. 1996;45:12-23.

10. Yu JR, Seo M, Kim YW, Oh MH, Sohn WM. A human case of gastric infection by Pseudoterranova decipiens larva. Korean J Parasitol. 2001;39:193-6.

11. Umehara A, Kawakami Y, Ooi HK, Uchida A, Ohmae H, Sugiyama H. Molecular identification of Anisakis type I larvae isolated from hairtail fish off the coasts of Taiwan and Japan. Int J Food Microbiol. 2010;143:161-5.

12. Mattiucci S, Fazii P, De Rosa A, Paoletti M, Megna AS, Glielmo A, et al. Anisakiasis and gastroallergic reactions associated with Anisakis pegreffii infection, Italy. Emerg Infect Dis. 2013;19:496-9.

13. Qin Y, Zhao Y, Ren Y, Zheng L, Dai X, Li Y, et al. Anisakiasis in China: the first clinical case report. Foodborne Pathog Dis. 2013;10:472-4.

14. Kim J-S, Oh K-S, Lee J-S. Comparison of food component between conger eel (Conger myriaster) and sea eel (Muraenesox cinereus) as a sliced raw fish meat. J Korean Fish Soc. 2001;34:678-84.

15. Choi P, Hur JW, Lim JH, Lee JY, Kim DW, Park MI, et al. A case of gastric submucosal tumor suspected to be caused by Anisakis. Korean J Gastrointest Endosc. 2003;27:26-30.

16. Uchida K, Nishihara M, Shinanba T, Yoshoda T, Miyamoto Y, Kakihara T. Behavioral observations of white spotted conger eel Conger myriaster in baited traps in Tokyo Bay. Proceedings of the Design Symposium on Conservation of Ecosystem (The 12th SEASTAR 2000 Workshop). Kyoto: Kyoto University; 2013. p. 109-14.

17. Park J-Y, Cho H, Kang J-H, Kim E-M, An C-M, Kim J-H, et al. Development of DNA microarray for species identification of eels (Anguilliformes and Myxiniformes) in Korean fisheries markets. Bio Chip J. 2014;8:310-6.

18. Froese R, Pauly D, editors. FishBase. World Wide Web electronic publication. http://www.fishbase.org, version. 2017:02. Accessed 12 Nov 2017.

19. Tang Y-M, Wu C-W. On biologic habits and resources distribution of common Japanese conger Conger myriaster (Brevoort). J Zhejiang Coll Fish. 1988;7:19-26.

20. Yang B-Z, Tang $Y-L$, Liang Z-L. Selectivity of escape-hole size in tube traps for white-spotted conger Conger myriaster. J Ocean Limnol. 2011;29:1041-7.

21. Li L, Zhao W-T, Guo Y-N, Zhang L-P. Nematode parasites infection in the starry batfish Halieutaea stellata (Vahl) (Lophiiformes: Ogcocephalidae) from the East and South China Sea. J Fish Dis. 2016;39:515-29.

22. Zhao W-T, Lü L, Chen H-X, Yang Y, Zhang L-P, Li L. Ascaridoid parasites infecting in the frequently consumed marine fishes in the coastal area of China: a preliminary investigation. Parasitol Int. 2016;65:87-98.

23. Berland B. Nematodes from some Norwegian marine fishes. Sarsia. 1961;2:1-50.

24. Smith JW. Larval Anisakis simplex (Rudolphi, 1809, det. Krabbe, 1878) and larval Hysterothylacium sp. (Nematoda: Ascaridoidea) in euphausiids (Crustacea: Malacostraca) in the North-East Atlantic and northern North Sea. J Helminthol. 1983;57:167-77.

25. Li L, Liu Y-Y, Zhang L-P. Morphological and genetic characterization of Hysterothylacium zhoushanensis sp. nov. (Ascaridida: Anisakidae) from the flatfish Pseudorhombus oligodon (Bleeker) (Pleuronectiformes: Paralichthyidae) in the East China Sea. Parasitol Res. 2012;111:2393-401.

26. Shamsi S, Gasser RB, Beveridge I. Description and genetic characterisation of Hysterothylacium (Nematoda: Raphidascarididae) larvae parasitic in Australian marine fishes. Parasitol Int. 2013;62:320-8.

27. Guo Y-N, Xu Z, Zhang L-P, Hu Y-H, Li L. Occurrence of Hysterothylacium and Anisakis nematodes (Ascaridida: Ascaridoidea) in the Tanaka's snailfish Liparis tanakae (Gilbert \& Burke) (Scorpaeniformes: Liparidae). Parasitol Res. 2014;113: $1289-300$

28. Zhu X, Gasser RB, Podolska M, Chilton NB. Characterisation of anisakid nematodes with zoonotic potential by nuclear ribosomal DNA sequences. Int J Parasitol. 1998;28:1911-21.

29. Chen $\mathrm{H}-\mathrm{Y}$, Shih H-H. Occurrence and prevalence of fish-borne Anisakis larvae in the spotted mackerel Scomber australasicus from Taiwanese waters. Acta Trop. 2015;145:61-7.

30. Tamura K, Stecher G, Peterson D, Filipski A, Kumar S. MEGA6: Molecular Evolutionary Genetics Analysis version 6.0. Mol Biol Evol. 2013;30:2725-9.
31. Posada D, Crandall KA. Selecting the best-fit model of nucleotide substitution. Syst Biol. 2001;50:580-601.

32. Felsenstein J. Confidence limits on phylogenies: an approach using the bootstrap. Evolution. 1985;39:783-91.

33. Hillis D, Bull MJJ. An empirical test of bootstrapping as a method for assessing confidence in phylogenetic analysis. Syst Biol. 1993;42:182-92.

34. Chai J-Y, Cho S-R, Kook J, Lee S-H. Infection status of the sea eel (Astroconger myriaster) purchased from the Noryangjin fish market with anisakid larvae. Korean J Parasit. 1992;30:157-62.

35. Li L, Zhao J-Y, Chen H-X, Ju H-D, An M, Xu Z, Zhang L-P. Survey for the presence of ascaridoid larvae in the cinnamon flounder Pseudorhombus cinnamoneus (Temminck \& Schlegel) (Pleuronectiformes: Paralichthyidae). Int J Food Microbiol. 2017;241:108-16.

36. Setyobudi E, Jeon CH, Choi K, Lee SI, Lee Cl, Kim J-H. Molecular identification of anisakid nematodes third-stage larvae isolated from common squid (Todarodes pacificus) in Korea. Ocean Sci J. 2013;48:197-205.

37. Zhang L-P, Du X-J, An R-Y, Li L, Gasser RB. Identification and genetic characterization of Anisakis larvae from marine fishes in the South China Sea, using an electrophoretic-guided approach. Electrophoresis. 2013;34: 888-94.

38. Pekmezci G-Z, Onuk E-E, Bolukbas C-S, Yardimci B, Gurler A-T, Acici M, et al. Molecular identification of Anisakis species (Nematoda: Anisakidae) from marine fishes collected in Turkish waters. Vet Parasitol. 2014;201:82-94.

39. Cavallero S, Magnaboscob C, Civettini M, Boffo L, Mingarelli G, Buratti P, et al. Survey of Anisakis sp. and Hysterothylacium sp. in sardines and anchovies from the North Adriatic Sea. Int J Food Microbiol. 2015;200:18-21.

40. Kong Q-M, Fan L-F, Zhang J-H, Akao N, Dong K-W, Lou D, et al. Molecular identification of Anisakis and Hysterothylacium larvae in marine fishes from the East China Sea and the Pacific coast of central Japan. Int J Food Microbiol. 2015;199:1-7.

41. D'Amelio S, Mathiopoulos KD, Brandonisio O, Lucarelli G, Doronzo F, Paggi L. Diagnosis of a case of gastric anisakidosis by PCR-based restriction fragment length polymorphism analysis. Parassitologia. 1999;41:591-3.

42. Paggi L, Mattiucci S, D'Amelio S. Allozyme and PCR-RFLP markers in anisakid nematodes, aethiological agents of human anisakidosis. Parassitologia. 2001; 43:21-7.

43. Fumarola L, Monno R, lerardi E, Rizzo G, Giannelli G, Lalle M, et al. Anisakis pegreffi etiological agent of gastric infections in two Italian women. Foodborne Pathog Dis. 2009;6(9):1157.

44. Mattiucci S, Paoletti M, Borrini F, Palumbo M, Palmieri RM, Gomes V, et al. First molecular identification of the zoonotic parasite Anisakis pegreffii (Nematoda: Anisakidae) in a paraffin-embedded granuloma taken from a case of human intestinal anisakiasis in Italy. BMC Infect Dis. 2011;11:82.

45. Lim H, Bong-Kwang J, Cho J, Yooyen T, Shin EH, Chai JY. Molecular diagnosis of cause of anisakiasis in humans, South Korean. Emerg Infect Dis. 2015;21:342-4.

46. Du C-X, Zhang L-P, Shi M-Q, Ming Z, Hu M, Gasser RB. Elucidating the identity of Anisakis larvae from a broad range of marine fishes from the Yellow Sea, China, using a combined electrophoretic-sequencing approach. Electrophoresis. 2010;31:654-8.

47. Mattiucci S, Nascetti G. Molecular systematics, phylogeny and ecology of anisakid nematodes of the genus Anisakis Dujardin, 1845: an update. Parasite. 2006;13:99-113.

48. Mattiucci S, Nascetti G. Advances and trends in the molecular systematics of anisakid nematodes, with implications for their evolutionary ecology and host-parasite co-evolutionary processes. Adv Parasitol. 2008;66:47-148.

49. Zhang L-P, Hu M, Shamsi S, Beveridge I, Li H-M, Xu Z, et al. The specific identification of anisakid larvae from fishes from the Yellow Sea, China, using mutation scanning-coupled sequence analysis of nuclear ribosomal DNA. Mol Cell Probe. 2007;21:386-90.

50. Li J, Guo J-N, Zhou J-B, Shi W, Li W-W, Fang F, et al. Preliminary investigation of Anisakis sp. third stage larvae infection of Pneumatophorus japonicus from the Yellow Sea area, China. Chinese J Food Hyg. 2013;25:5661.

51. Šimat V, Miletić J, Bogdanović T, Poljak V, Mladineo I. Role of biogenic amines in the post-mortem migration of Anisakis pegreffii (Nematoda: Anisakidae) larvae into fish fillets. Int J Food Microbiol. 2015;214:179-86.

52. Cipriani P, Acerra V, Bellisario B, Sbaraglia GL, Cheleschi R, Nascetti G, et al. Larval migration of the zoonotic parasite Anisakis pegreffii (Nematoda: Anisakidae) in European anchovy, Engraulis encrasicolus: Implications to seafood safety. Food Control. 2016;59:148-57. 
53. Shih $\mathrm{H}-\mathrm{H}$, Ku C-C, Wang C-S. Anisakis simplex (Nematoda: Anisakidae) thirdstage larval infections of marine cage cultured cobia, Rachycentron canadum L., in Taiwan. Vet Parasitol. 2010;171:277-85.

54. Arai T, Akao N, Seki T, Kumagai T, Ishikawa H, Ohta N, et al. Molecular genotyping of Anisakis larvae in middle eastern Japan and endoscopic evidence for preferential penetration of normal over atrophic mucosa. PLoS One. 2014;9:e89188.

55. KØie M. Aspects of the life-cycle and morphology of Hysterothylacium aduncum (Rudolphi, 1802) (Nematoda, Ascaridoidea, Anisakidae). Can J Zool. 1993;71:1289-96.

56. Klimpel S, Rückert S. Life cycle strategy of Hysterothylacium aduncum to become the most abundant anisakid fish nematode in the North Sea. Parasitol Res. 2005;97:141-9.

57. Li L, Gibson DI, Zhang L-P. An annotated catalogue of the ascaridoid nematode parasites of Chinese vertebrates. Syst Parasitol. 2016;93:1-35.

58. Najjari M, Sadjjadi SM, Derakhshanfar A, Ebrahimipour M. Hysterothylacium amoyense in Platycephalus indicus: a Persian Gulf fish and its experimental infection of mouse model. Comp Clin Pathol. 2016;25:1143-9.

59. Sasal P, Niquil N, Bartoli P. Community structure of digenean parasites of sparid and labrid fishes of the Mediterranean Sea: a new approach. J Parasitol. 1999;119:635-48.

60. Choi JH, Choi SH, Kim JB, Park J-H, Oh CW. Feeding ecology of the whitespotted conger eel (Conger myriaster) in the Southern Sea of Korea. J Korean Fish Soc. 2008:41:282-8.

61. Huss HH, Embarek PKB. Assessment and management of seafood safety and quality. FAO Fisheries Technical Paper. 2004;444:60-70.

Ready to submit your research? Choose BMC and benefit from:

- fast, convenient online submission

- thorough peer review by experienced researchers in your field

- rapid publication on acceptance

- support for research data, including large and complex data types

- gold Open Access which fosters wider collaboration and increased citations

- maximum visibility for your research: over $100 \mathrm{M}$ website views per year

At BMC, research is always in progress.

Learn more biomedcentral.com/submissions 